\title{
Integrated Design Approach for Shell Structures Using Isogeometric Analysis
}

\author{
Philipp Längst ${ }^{1}$ (D) Alexander Michalski ${ }^{1}$ • \\ Julian Lienhard ${ }^{1}$
}

\begin{abstract}
Shell and tension surface structures are characterized by complex curved geometries with a potential for structural efficiency and formal quality. To unveil this potential, it is essential to define, modify and analyse complex geometries in an integral way. Describing complex, free-form geometries, within a computer-based design process, non-uniform rational B-Splines (NURBS)-based methods can be considered as standard within the common working process. The mathematical description of geometry with NURBS within a finite element-based analysis has become known as isogeometric analysis (IGA). As the basic descriptions of design and analysis models are equivalent, transforming steps (such as "meshing", etc.) become irrelevant, and it is possible to fully unify the design and analysis environment. For the direct integration of isogeometric-based structural analysis into the design process the authors developed an interface for the computer aided design (CAD) environment that can directly access a broad field of analysis tasks (such as structural analysis, form-finding, etc.). As a result, the designing architect or engineer is capable of instantly estimating the structural qualities of arbitrary geometries. The integration of architectural and structural design by IGA with CAD enables an integrated design approach. This will be demonstrated in the context of the design of shell structures. To support the design process, the interface contains tools to provide important values such as strain energy to, for example, evaluate the structural efficiency of a random shell geometry within an early design stage. This strongly supports the designer to meet different design criteria derived from either structural or formal aspects. After a brief introduction of the isogeometric analysis method, this paper will highlight the potentials of this method through a variety of case studies. In each study both engineering and architectural design aspects will be
\end{abstract}

Philipp Längst

laengst@str-ucture.com

1 str.ucture GmbH, Lindenspürstr. 32, 70176 Stuttgart, Germany

Birkhäuser 
addressed by comparing the process and the results of finite element analysis, and highlighting the integrated variability of design.

Keywords Shell structures · Form-finding · Isogeometric analysis (IGA) · Integrated design approach · Direct shell evaluation

\section{Introduction}

Shell structures are amongst the most efficient types of structures in the ratio of large span to self-weight. They carry external loads via a combination of axial tension, compression and shear stress without significant bending components. Therefore, they are classified as surface-active lightweight structures.

The typical structural characteristics of a shell mainly result from a symbiosis of geometry and boundary conditions (Williams 2014). Consequently, defining the shell geometry is an essential part of the design process. The procedure for developing the geometry of lightweight structures (such as shells) has become known as form-finding (Lienhard 2016). Generally, form-finding is based on the principal that form is dictated by the flow of forces. It can be described as a deterministic process in which a setup of physical boundary conditions leads to a geometry which is previously un-known. For example, the designer defines a target distribution of internal stress, supports and a loading situation. Form-finding methods then aim to find a geometry that will carry the given load with the defined distribution of internal stress. This can be described as an inversion of conventional structural design processes, where the geometry mainly is an outcome of architectural constraints.

From the perspective of lightweight engineering, computational-based formfinding has become a common design tool. Still, from the perspective of architecture form-finding-based design is far more complex. Firstly, because the designer is only able to control the resulting geometry via the setup of starting conditions. As a consequence, the final shape, and its aesthetic appearance in particular, can only be influenced implicitly. Secondly, structural efficiency does not inherently implicate aesthetic quality. In addition, it is often difficult to define criteria that consider aesthetically related conditions within the mathematical problem formulation of form-finding. If possible, the criteria need to be translated into explicit conditions i.e. a specific ratio of curvature to total span widths.

A modern approach to lightweight engineering seeks therefore to meet structural as well as architectural requirements. In terms of form-finding this means that form may be informed rather than dictated by structural criteria. One may ask for a design result that is very close to an optimum of structural efficiency but at the same time fulfils specific architectural conditions, e.g. a certain aesthetic appearance. Regarding structural aspects, the capability of computational analysis methods is required. On the contrary, insignificant changes to architectural conditions can have a significant effect on the structural efficiency. In this context, particularly, the potential of parametric-based architectural design should be considered. 
This calls for new design techniques and design environments that exploit the full potential of both computational analysis and computational parametric-based design. This enables tribute to be paid to the multiple parameters of design from the very beginning. Bringing together simulation and design environments can be considered as a significant step. In the present scenario, completely different mathematical geometry descriptions can be seen as a major handicap. However, promising concepts regarding a new type of finite element analysis (FEA), called isogeometric analysis (IGA) as presented by Cottrell et al. (2009) or Breitenberger et al. (2015), have been introduced in the literature.

In this paper a new integrated approach in lightweight design and its application will be presented. The focus is on using a new way of combining IGA-based analysis methods with parametric-based design processes. Hence, it directly integrates the simulation into the design environment. This allows for a consistent way of defining, modifying and developing structural objects within the design and simulation process.

\section{Isogeometric Analysis}

Currently, the typical design processes in architecture and structural engineering use separate software environments. Computer aided design (CAD) software is used to create computational geometry models. To perform structural analysis, mainly computer aided engineering (CAE) software containing finite element solvers is used. From a mathematical perspective, the data models in CAD (geometric model), and CAE (analysis model), are based on completely different representations. In $\mathrm{CAD}$ a geometric object is described by non-uniform rational B-splines (NURBS)based parametrizations. In CAE, mainly isoparametric finite element models are used to describe the geometry. As a result, certain methods are required to convert one model having a particular mathematical description into another mathematical description (changing the parametrization). Meshing the CAD geometry for analysis is a common example. Translating methods are often time-consuming, especially within an iterative design process. Therefore, they often become the bottleneck in a computer-aided design process (Cottrell et al. 2009). This also applies for reverse methods. As a consequence, the inclusion of analysis results in CAD is mostly unknown in common practice.

Isogeometric analysis (IGA), as introduced by Cottrell et al. (2009), aims at unifying the different mathematical worlds that are described above. It provides an identical mathematical representation of CAD and CAE models. This is achieved by using NURBS to discretize the deformation within a displacement-based finite element analysis. As a result, model transformations such as meshing become redundant, as shown in Fig. 1. The models only differ in terms of the fineness of parametrization, meaning, that is, a higher amount of control points. This is required by the analysis model to approximate the solution with reasonable accuracy.

The isogeometric B-REP analysis (IBRA), introduced by Breitenberger et al. (2015), can be described as a generalization of IGA. The boundary representation (B-Rep) is a topological form of representing NURBS objects. For 2D-NURBS 


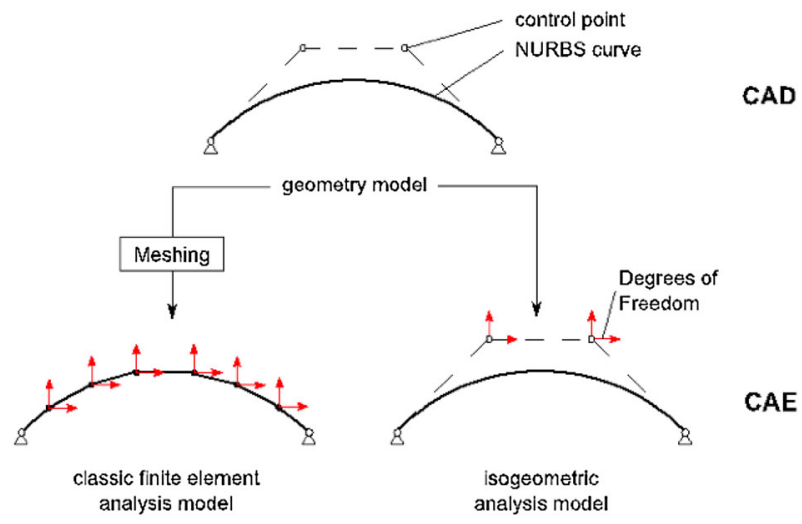

Fig. 1 Analysis models for classic finite element and isogeometric analysis derived from the same geometry model: for classic FEA, the model needs to be transformed (meshed), while for IGA the geometry does not need to be transformed. The degrees of freedom are located differently on both of the models. Graphic: author

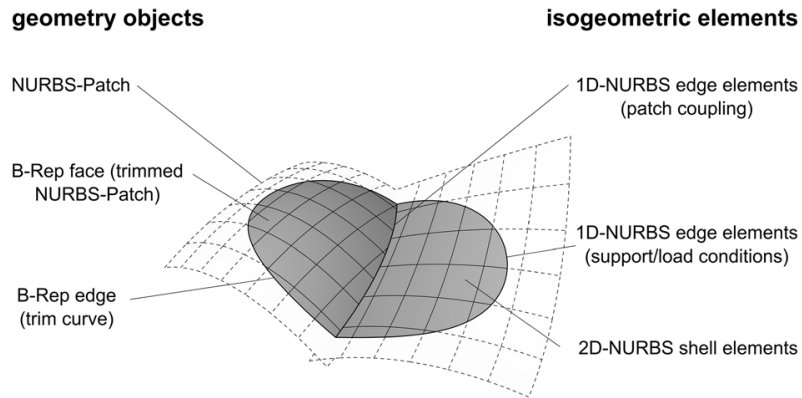

Fig. 2 Discretization of a geometry model, consisting of two arbitrarily trimmed and parametrized NURBS-patches according to their B-Rep topology for IBRA: 2D-elements are used for faces, while 1Delements for edges, which also allow for definition of boundary conditions and coupling. Graphic: author

objects, the B-Rep description distinguishes between the object's faces, edges and vertices. In terms of the finite element analysis of shells, IBRA-based elements allow the geometry to be discretized respectively: faces are discretized using 2DIBRA-elements and edges using 1D-IBRA-elements. Face and edge elements are mutually coupled. Boundary conditions can be applied to edge or surface elements. Edge elements can also be used for coupling with other patches.

Consequently, IBRA allows for a uniform and consistent way of discretizing a geometry object that consists of arbitrarily trimmed, coupled and parametrized 1Dand 2D-NURBS-patches (see also Fig. 2). As a result, the CAD geometry does not need modification when used for analysis. This can be considered as a key aspect in terms of application.

Furthermore, NURBS-based finite elements enable direct access to state-of-theart simulation techniques (non-linear analysis, form-finding or optimization). Also, the analysis results can directly be linked back to CAD to visualize results i.e. deformations. 


\section{Designing Shell Structures with an Integrated Approach}

The integration of IGA or IBRA into the computational design environment of CAD enables simulation results to immediately influence design decisions. This will be referred to as an integrated design approach, because it allows the designer to respond to architectural as well as structural needs at the same time. With respect to the computational design procedure, the following aspects are fulfilled:

- A consistent geometric representation is used throughout the entire design process.

- Different types of simulations can be performed and their results are directly displayed in CAD. The software and design environment do not need to be changed.

- Simulation results (e.g. a geometry which is the result of a numerical formfinding) can be modified in the common CAD design manner and reanalyzed.

Figure 3 illustrates the design process of the research pavilion M1, built in Monthoiron, France, in 2011 (Lienhard and Knippers 2015). The pavilion can be described as a bending-active textile hybrid structure with an integrated tensile membrane.

For the form-finding and design classic $\mathrm{CAD} / \mathrm{CAE}$ was used. The structural model was developed step-wise by cumulative addition of results from all the previous form-finding steps in the current step (incremental form-finding). This was done in order to consider the different internal stress conditions of the entire design process. The steps are listed in the following paragraph.

In steps $1-3$, the geometry of the bending rods is determined. Straight beam elements were used as initial geometry. In step 4, a meshed surface object is added, using the final rod geometry as its boundary. It serves as the input geometry for form-finding the membrane spanning between the already form-found rods. The final structure, including the form-found membrane, is shown in step 5.

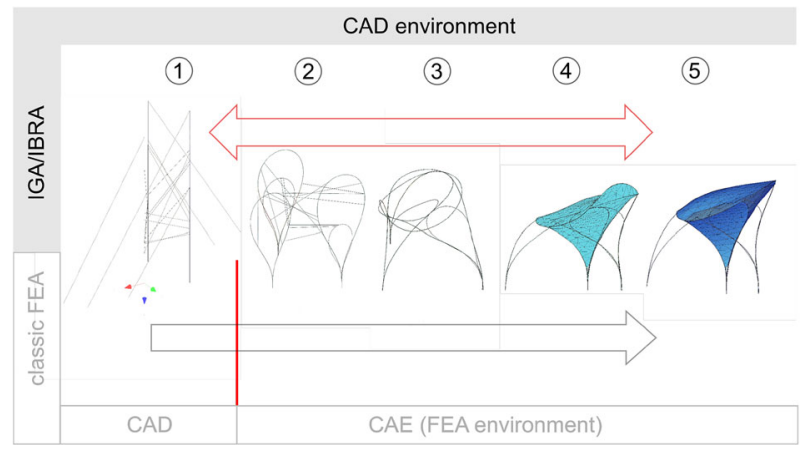

Fig. 3 Design of a bending active structure. In contrast to a classic CAD/CAE process, IGA-based design allows for geometric modifications at any stage; (1) definition of start geometry of rods, (2) formfinding of rods, (3) bend form-found rods in defined place, (4) definition of start geometry of membrane, (5) form-finding of membrane. Graphic: author 


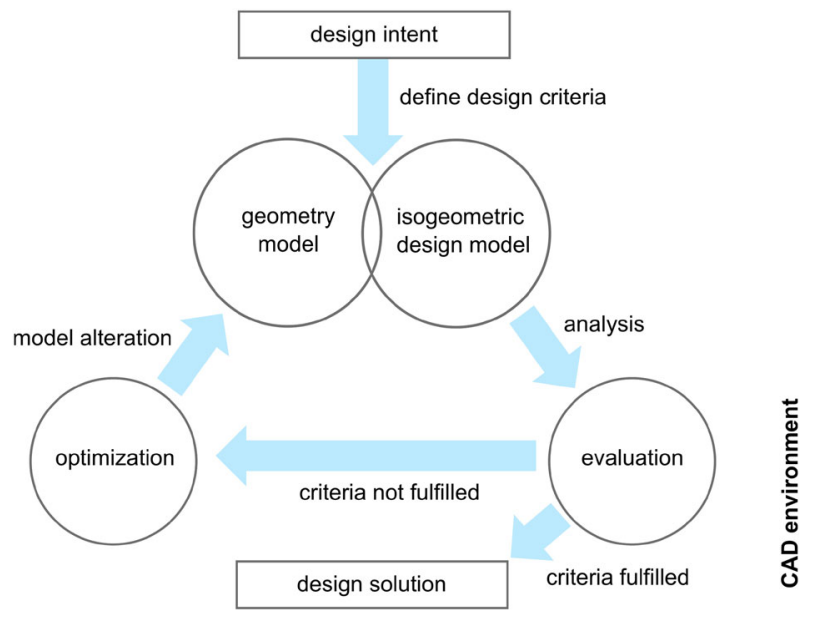

Fig. 4 Design sequence of shell structure within CAD, based on an integrated approach. Graphic: author

This example illustrates the differences between integrated and common design procedures. Integrated isogeometric-based simulation enables the designer to modify intermediate states of design at any time. In the classic FEA-based procedures, CAD is only used to define the starting geometry. Any modifications in the intermediate geometries (e.g. add new geometry objects as shown in step 4 of Fig. 3), must be carried out within the CAE environment which usually does not provide the relevant tools. In terms of application, this procedure is often inconvenient and prone to errors.

The integrated approach has a significant impact on the design of shell structures. Already in the early stages of design, the main focus is often on exploring different design shapes. In this context an evaluation concept is presented here. It aims to evaluate a shell geometry regarding its load-bearing properties and structural integrity directly within $\mathrm{CAD}$, using IGA/IBRA results. As a result, multiple different shell geometries can be compared easily. This shall support the process of finding a geometry that sufficiently fulfills the design intent defined by the architect as well as the engineer.

In Fig. 4, the application of the evaluation concept is shown within a typical design sequence. In a first step, the geometry and the decisive design criteria for shape and structural behavior are defined. The isogeometric analysis model is directly derived from the geometry model. In a second step, a structural analysis is performed and its results are shown in the CAD environment. This allows the shell geometry to be evaluated with respect to the set design criteria. If the criteria cannot be fulfilled, the geometry model (and analysis model respectively) needs to be modified, analyzed and re-evaluated. The modifications of the geometry model can include manual changes as well as numerical procedures and optimization techniques.

For the evaluation of shell structures, two primary criteria are considered:

- Load-bearing capacity: 
Can the shell withstand a given load? This includes a check on stress and stability.

- Load-bearing behavior:

How does bending and membrane action contribute to load transfer? For membrane action, each fiber in the shell cross-section is equally stressed (membrane state), while bending action results in an inhomogeneous stress distribution with high concentration on the top and bottom surface (bending state).

Strain energy plays a key role in the evaluation of the load-bearing behavior of a shell. In general, the load transfer via membrane action corresponds to much more rigid structural behavior than the load transfer due to bending. As a result of the thin shell section, its bending stiffness is usually negligibly small compared to its axial stiffness. Inextensional deformations ${ }^{1}$ can occur when shells are exposed to systematic bending (Williams 2014). Consequently, shells should primarily be in a membrane state thus showing a rigid load-bearing behavior.

The strain energy is influenced by the stress and strain of a section. As given in Timoshenko and Goodier (1951), it is calculated by integrating the product of stress $\sigma$ with strain $\varepsilon$ over the shell thickness $t$. This will be referred to as local strain energy $w_{\text {int }}$ in the following,

$$
w_{i n t}=\frac{1}{2} \int_{t} \sigma \varepsilon d t
$$

The integration over the total domain of the structure $\Omega$ provides the global strain energy $W_{\text {int }}$

$$
W_{i n t}=\int_{\Omega} w_{i n t} d \Omega .
$$

Loads that cause bending deformations will also cause much higher strain energy, compared to loads that cause predominantly membrane action. This is due to the significant difference in bending and axial stiffness. Therefore, designing a rigid shell with maximized stiffness corresponds to the minimization of the total strain energy (Bletzinger and Ramm 2014). For a given structure and loading, the minimum total strain energy will occur when the structure is in a pure membrane state. In effect, one may look at the ratio of strain energy, resulting from bending only, to the total strain energy, in order to evaluate the structural stiffness.

The local strain energy resulting from bending $w_{\text {int }, m}$ is calculated with stresses $\sigma_{m}$ due to bending moment $m$ and the respective strain $\varepsilon_{m}$. This is equivalent to $m$ times the section's curvature $\kappa$

$$
w_{\text {int }, m}=\frac{1}{2} \int_{t} \sigma_{m} \varepsilon_{m} d t=\frac{1}{2} m \kappa .
$$

The ratio $\eta_{w}$ of strain energy, resulting from bending, to the total strain energy is then calculated as

\footnotetext{
${ }^{1}$ Large deformations, where the shell is bent without stretching.
} 


$$
\eta_{w}=\frac{w_{\text {int }, m}}{w_{\text {int }}}=\frac{w_{\text {int }, m}}{w_{\text {int }, n}+w_{\text {int }, m}}
$$

where $w_{i n t, n}$ is the strain energy, resulting from membrane action.

Equation 4 gives information about the stiffness state of a structure. For a ratio close to zero a rather rigid structural behavior can be assumed. The strain energy resulting from bending in comparison to the total strain energy is relatively small. A ratio close to 1.0 indicates a bending-dominated load-transfer, which is linked to a rather soft structural behavior.

The ratio $\eta_{W}$ is the equivalent ratio, considering the global strain energy and its portions due to membrane and bending state. It enables the designer to compare various shell structures by a single value, which is convenient in terms of direct evaluation or ranking. Additionally, it provides information about the structural efficiency of a shell. Sections, predominantly exposed to membrane action, experience a uniform stress distribution. This leads to a consistent and efficient material utilization. In contrast, bending-dominated strain leads to high stress at the top and bottom of the section, while the inner section stays unstressed.

It may be noticed that generally very rigid shell structures show higher tendency of failing due to stability problems. This leads to contradictory design targets. For detailed information taking into account further aspects of shell structure evaluation refer to Längst (2015).

\section{Integration of IBRA into CAD}

An interface was developed to directly access IBRA by integrating a pre- and postprocessing control into the CAD environment. As shown in Fig. 5 the interface transfers geometry and analysis information to an external finite element solver and incorporates analysis results back into the CAD environment. This includes the following main features:

- Discretization of NURBS geometry model by assignment of properties (e.g. materials, sections) and boundary conditions (e.g. support conditions, loading) to geometric objects directly in CAD.

- Visualization of results in CAD viewport (deformed structure as NURBS geometry with the result plotted onto it).

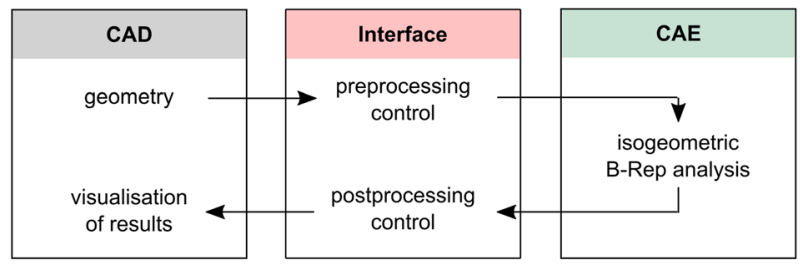

Fig. 5 Interface for CAD, which links CAE- with CAD environment: geometry and analysis information are transferred to an external finite element program, performing IBRA, and results are visualized in CAD environment. Graphic: author 


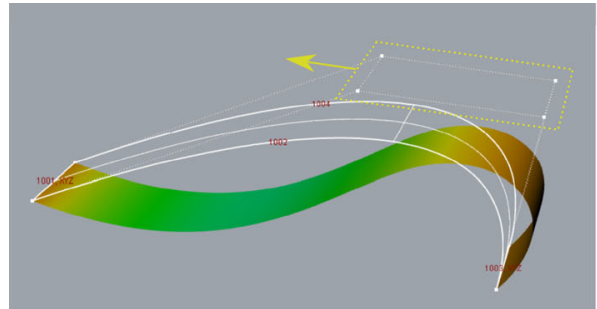

(a)

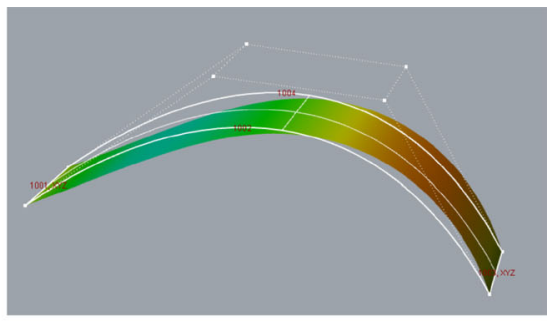

(b)

Fig. 6 Direct visualization of geometry update impact on deflection results of a single-curved shell subjected to self-weight: a the NURBS geometry is altered by moving the control points; b new geometry: the deformed shape of the new geometry is updated automatically by performing an analysis update in the background. Graphic: author

The interface was developed for the CAD-software Rhinoceros ${ }^{\circledR} 5.0$ (McNell and Associates) using the visual scripting extension Grasshopper ${ }^{\circledR}$ in combination with scripting languages Python and C\#. Grasshopper ${ }^{\circledR}$ contains automated script-update functionalities. This allows it to visualize the impact of changes to geometry or analysis variables (e.g. material properties or loading) in real-time. For performing IBRA the finite element program CARAT ++ is used, which was developed by the Chair of Structural Analysis, Prof. Dr.-Ing. K.-U. Bletzinger, Technical University of Munich.

An example of the application is illustrated in Fig. 6. It shows a single curved shell geometry, for which the deflections under self-weight are simulated, using IBRA. Via the interface, the external solver is accessed and the deformed shape visualized. For the first design intent (see Fig. 6a) significant deflections are detected. The geometry is then altered in order to reduce the deflections. This is done by moving the control points of the NURBS-surface in what can be considered as a standard CAD-operation. The geometric modification automatically calls for an update of the analysis tasks and its impact on the deflection results is directly visualized (see Fig. 6b).

Referring back to the evaluation concept introduced in the previous section, the interface also includes a respective mode of result visualization, which is shown in Fig. 7. It provides the essential results at the same time, plotted in separate "boxes".

In box (2), the results to evaluate the bearing capacity are given. Box (3) shows results for load-transfer evaluation, such as the ratio of strain energy. In box (4) result plots for membrane forces and bending moments are shown.

\section{Case Study}

In this section a case study is described. It deals with the design of the shape of a concrete shell by using the integrated approach and the software interface introduced in the previous section. The design intent is to fulfil the architectural as well as the physical criteria of a lightweight shell structure. 


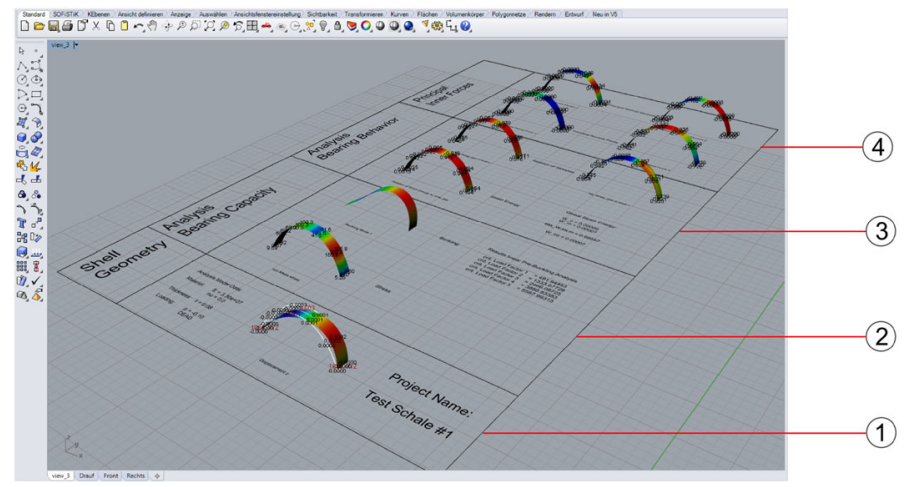

Fig. 7 Result display layout in CAD-viewport to provide relevant information for shell evaluation: (1) design geometry, (2) evaluation of load-bearing capacity, (3) evaluation of load-bearing behaviour, (4) internal forces. Graphic: author

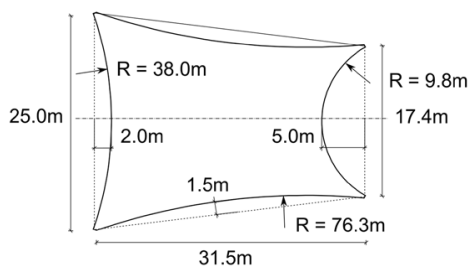

(a)

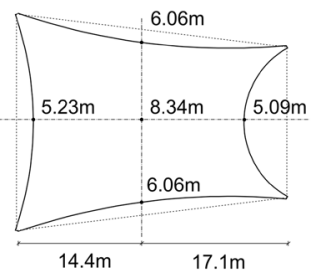

(b)

Fig. 8 Dimensions of concrete shell by H. Isler in Bex, Switzerland, based on Gallenmüller (1999); a dimension of projections of shell edges, $\mathbf{b}$ height of midpoints of edges and centre. Graphic: author

The concrete shell roof, designed by Heinz Isler, for the laboratory and research facilities of Gips Union SA in Bex, Switzerland, is used as a reference case. The shell is symmetric to one axis and rests on four corners. Its basic dimensions are taken from Gallenmüller (1999) and illustrated in Fig. 8. In plan-view the shell spans $31.5 \mathrm{~m}$, and $25.0 \mathrm{~m}$ respectively, with a shell thickness of $8.0 \mathrm{~cm}$ (not taking into account the increased sections size close to the supports).

The following design criteria shall be met:

- Architectural:

The plan-view projection of the free edges, the height of the shells centre and the heights of the centres of the free edges are predefined (see Fig. 8).

- Structural:

The load transfer via bending action was supposed to be reduced to a minimum (considering the shell's self-weight). This is equivalent to obtaining maximum structural efficiency.

Starting from the given constraints, a doubly curved geometry was developed in CAD, using standard NURBS-operations. Thus the geometry of the shell was not mechanically motivated. The shell, which is shown in Fig. 9, was parametrized by a single NURBS patch. The patch corners were trimmed to create line-type supports. 


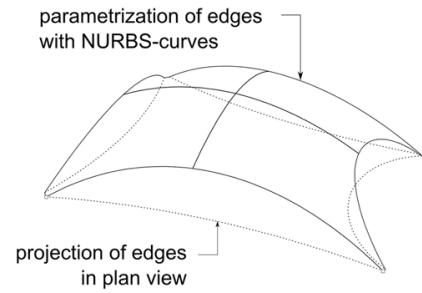

(a)

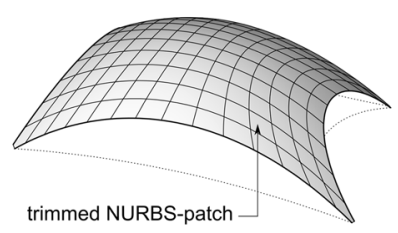

(b)

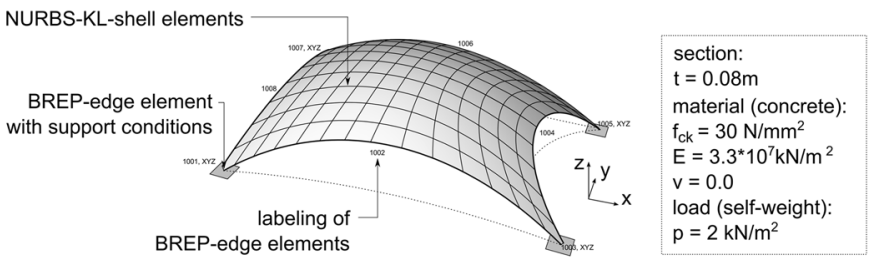

(c)

Fig. 9 Geometry and analysis model: a definition of edges with NURBS curves according to the given projection in plan-view and shell centre, $\mathbf{b}$ definition of NURBS-patch which contains edge curves (geometry model), c allocation of properties for IBRA analysis model. Graphic: author

To retrieve the analysis model from the geometry model, mechanical information (element types, support conditions, etc.) were assigned to the respective NURBS geometry objects, using the interface. In the context of the given example NURBSbased Kirchhoff-Love-shell elements were used. The interface also derived the NURBS-data, which was required to discretize the geometry. Finally, the input for an external linear finite element analysis was compiled.

The analysis results can be visualized in the CAD viewport as mentioned in the previous section and are shown in Fig. 10. The designer has instant access to key values and information concerning the structural behaviour of the shell geometry. The results for the given design intent are shown below and interpreted in order to evaluate the design.

The distribution of the von Mises-stress, given in Fig. 11a, shows a maximum stress of $38 \mathrm{~N} / \mathrm{mm}^{2}$ in areas close to the supports. This exceeds the design strength of concrete, which usually is around $15-20 \mathrm{~N} / \mathrm{mm}^{2}$. Therefore, it can be assumed that the required bearing capacity is not provided. As mentioned above, the increased section depth close to the supports was not considered in this context.

The local ratio of strain energy from bending to the total strain energy is given in Fig. 11b. It can be seen that the local ratio of strain energy is greater than 0.90 in most parts of the shell. This indicates a bending-dominated load bearing behaviour. The global strain energy leads to a similar impression. With global strain energy values of $\mathrm{W}_{\mathrm{int}, \mathrm{m}}=6.007 \mathrm{kNm}$ (bending action only) and $\mathrm{W}_{\mathrm{int}, \mathrm{n}}=1.804 \mathrm{kNm}$ (membrane action only) it follows 


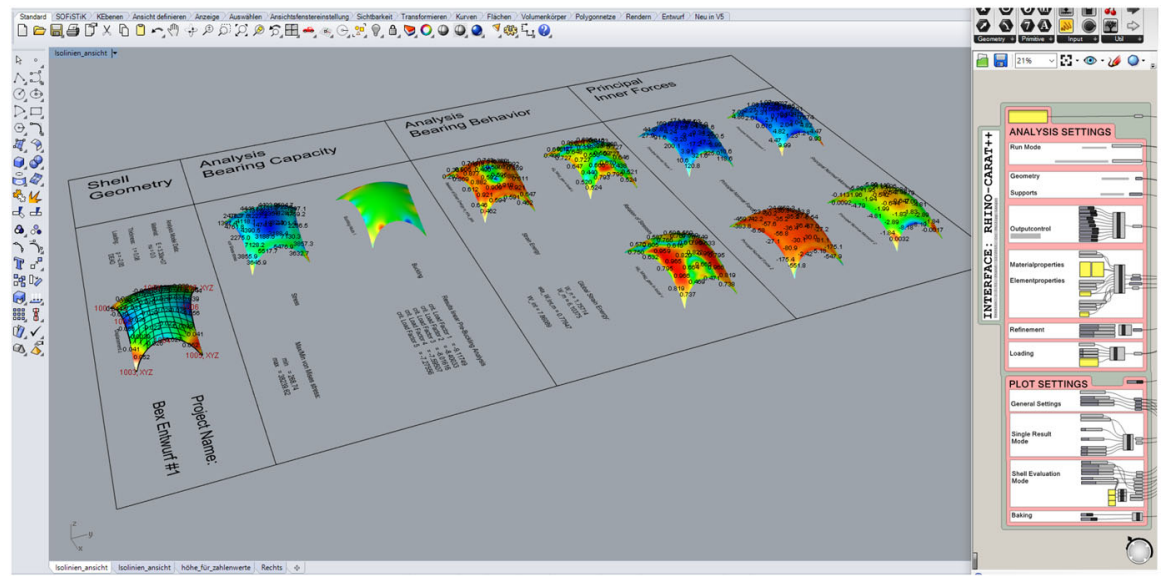

Fig. 10 Results of IBRA for the evaluation of case study shell geometry visualized in CAD-viewport. Graphic: author

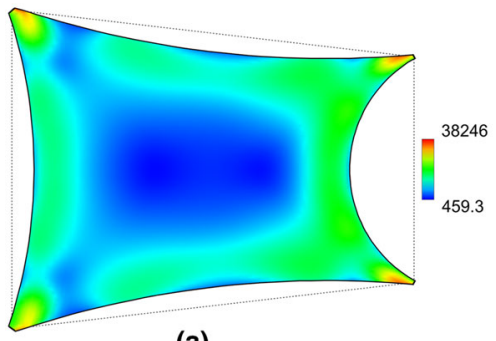

(a)

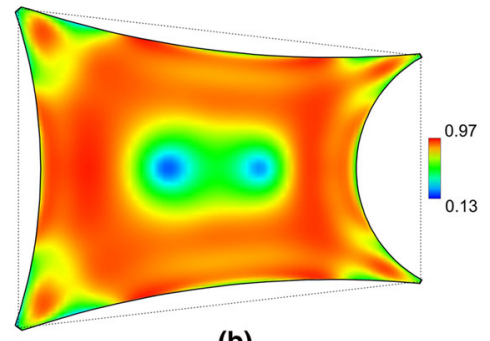

(b)

Fig. 11 Results for start geometry: a distribution of von Mises stresses in $\mathrm{kN} / \mathrm{m}^{2}$; b distribution of local ratio of strain energy. Graphic: author

$$
\eta_{W}=\frac{W_{i n t, m}}{W_{i n t, n}+W_{i n t, m}}=\frac{6.007}{1.804+6.007}=0.77 .
$$

The internal work resulting from bending action is almost three times higher than the respective value due to membrane action. As a result, the geometry can be considered as relatively soft and flexible. Large deformations can be expected, which may cause overstressing of the shell cross-section. Additionally, an inefficient structural behaviour can be assumed which calls for optimisation of the initial geometry.

To optimize the original geometry an approach similar to numerical hanging models was used. Numerical hanging models are known as one method for the numerical form-finding of shell structures (Bletzinger and Ramm 2014). The procedure can be described as follows. In a first step, the geometry was mirrored on the xy-plane. As a result, the self-weight causes mainly internal tensile stress. In a second step, the thickness of the shell was reduced by a factor of 0.1 , whereas the Young's modulus was increased by a factor of 10. This results in an artificial 


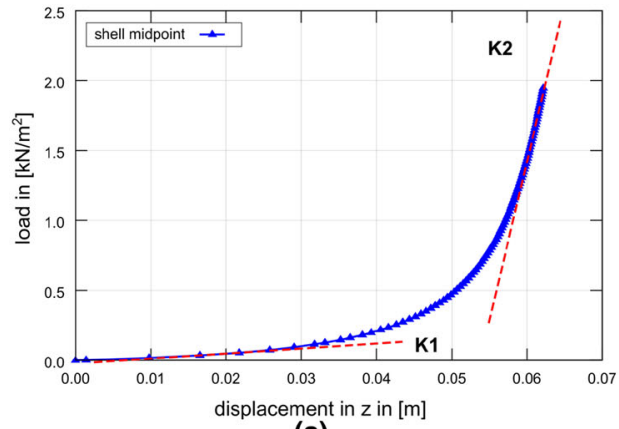

(a)

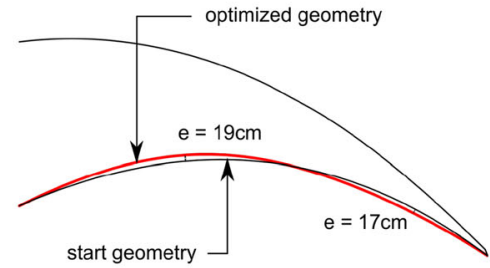

(b)

Fig. 12 a Load-displacement diagram of non-linear analysis for z-displacement of shell centre point; b edge comparison of original and optimized geometry. Graphic: author

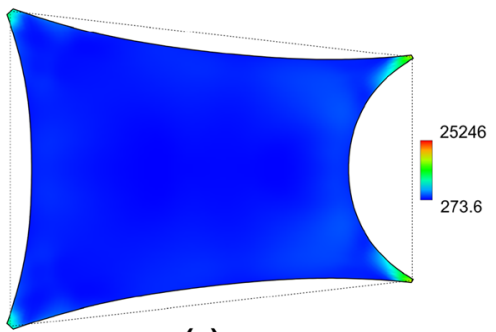

(a)

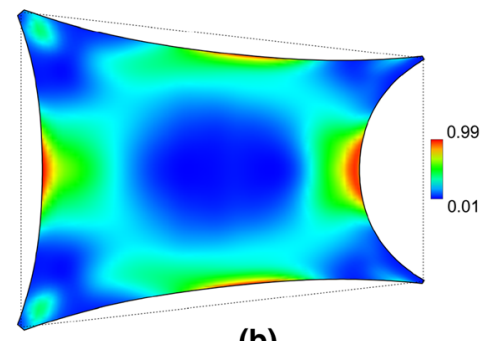

(b)

Fig. 13 Results for optimized geometry: a distribution of von Mises stresses in $\mathrm{kN} / \mathrm{m}^{2}$; $\mathbf{b}$ distribution of local ratio of strain energy. Graphic: author

reduction of bending stiffness while the membrane stiffness remains unaffected. As a result, the membrane action of forces is dominant and the total load-transfer behaviour becomes stiffer.

From the perspective of simulation, a geometrically non-linear problem was solved where the self-weight was applied gradually. Once the full self-weight is applied, the deformed shape (resultant geometry) can be seen as the new, optimized geometry. Figure 12a shows the tangential stiffness of the hanging model $\boldsymbol{K}$ at the beginning $(\boldsymbol{K} 1)$ and under full self-weight $(\boldsymbol{K} 2)$. The steep rise of the curve shows that the hanging model was "stiffened".

This resulting optimised geometry was then mirrored again on the xy-plane. The difference between the two models was comparatively small, as shown in Fig. 12b. As the optimization was performed using the isogeometric NURBS elements (isogeometric analysis model), the resultant geometry was also described by NURBS.

The new shell shape was then analysed again. The linear analysis results of the new shell geometry are given in Fig. 13. The distribution of the local strain energy ratio shows a significant reduction. Load transfer by bending action has fallen below $5 \%$ for the main areas of the shell. Also the global strain energy ratio shows a membrane-dominated and thus stiff load-bearing behaviour with $\eta_{W}=0.12$. This 
indicates that the structural efficiency has been significantly improved. Also the distribution of von Mises-stress has become more uniform over the entire shell.

\section{Conclusion}

Modern approaches to the design of lightweight structures call for a common design and simulation environment that can provide access to computational analysis as well as computational design. In this context, isogeometric-based analysis (IGA/ IBRA) may be considered as a key aspect. It allows for a consistent mathematical description of geometry across CAD and CAE environments and thus helps to close the gap between the architectural and engineering world.

In this paper a design approach was presented which incorporates IGA/IBRAbased finite element analysis in the CAD environment. This method is referred to as an integrated approach. It aims at directly influencing the architectural and structural design by using information that is retrieved from real-time structural analysis feedback. This provides promising potentials and advantages, especially for lightweight design, such as the design of shell structures, because structural analysis as a design tool can be part of the designer's toolbox already from the very beginning.

With respect to the design of shell structures, the authors presented a concept to evaluate the structural behaviour of shells. The ratio of strain energy due to bending to the total strain energy of a structure was considered as the key parameter for evaluation of structural efficiency. Additionally, an interface was introduced which actively links the CAD- and CAE-environments and also allows for live structural analysis feedback in CAD. To reveal the potentials of an integrated approach to design within application, a case study was presented. It dealt with the design of a concrete shell where predefined structural as well as architectural constraints were considered.

The introduction of CAE within the CAD environment using isogeometric-based FEA provides a first glimpse of its benefits for the design process, especially in the domain of lightweight design. Future work will focus on the introduction of initially stressed geometric objects to the CAD environment for different construction stages. This will enable tracking of internal stress developments during the design process, which is currently not common in practice.

Acknowledgements The work presented was part of a research visit to the Chair of Structural Analysis, Prof. Dr.-Ing. K.-U. Bletzinger, Technical University of Munich for the execution of a scientific thesis for which the author expresses his gratitude. The research is funded within the ZIM initiative of BMWI.

\section{References}

Bletzinger, K.-U. and E. Ramm. 2014. Computational Form Finding and Optimization. In: Shell Structures for Architecture, eds. S. Adriaenssens, P. Block, D. Veenendaal and C. Williams, 46-55. London: Routledge. 
Breitenberger, M., et al. 2015. Analysis in Computer Aided Design: Nonlinear Isogeometric B-Rep Analysis of Shell Structures. Computer Methods in Applied Mechanics and Engineering 284: 401-457.

Cottrell, J. Austin, Thomas J. R. Hughes, and Jurij J. Bazilevskij. 2009. Isogeometric Analysis: Toward Integration of CAD and FEA. Chichester: Wiley.

Gallenmüller, M. 1999. Numerische Formfindung von Flächentragwerken am Beispiel einer Schale von Heinz Isler. Diplom Thesis, Institute for Structural Mechanics, University of Stuttgart.

Längst, P. 2015. Erweiterung und Anwendung des isogeometrischen Designprozesses zur Untersuchung von Schalentragwerken. Master Thesis, Institute for Structural Mechanics, University of Stuttgart.

Lienhard, J. 2016. The Potentials of Parametrics in the Design of Modern Lightweight Structures. GAM. 2016, 40-55.

Lienhard, J. and J. Knippers. 2015. Bending-Active Textile Hybrids. Journal of the International Association for Shell and Spatial Structures. 56 (1): 37-48.

Timoshenko, S. and J.N. Goodier. 1951. Theory of Elasticity. New York: McGraw-Hill.

Williams, Chris. 2014. What is a Shell? In: Shell Structures for Architecture, eds. S. Adriaenssens, P. Block, D. Veenendaal and C. Williams, 21-31. London: Routledge.

Philipp Längst born in 1989, received a B.Sc. degree (2012) in Civil Engineer from the University of Stuttgart, Germany. In 2013 he was part of Foster + Partners, Structural Engineering Department, London, UK. He graduated from University of Stuttgart with a M.Sc. degree in Civil Engineer in 2015, which included a research visit to the Chair of Structural Analysis, Prof. Dr.-Ing. K.-U. Bletzinger, Technical University of Munich. His studies focus on linking architectural design with new technologies in finite element analysis in order to explore new approaches in lightweight design. Currently, he is part of str.ucture GmbH, Stuttgart as a Structural Engineer.

Alexander Michalski born in 1976, is a civil engineer with 13 years of profound experience in the design, analysis and construction of light-weight structures. Moreover, he is a former member of the Chair of Structural Analysis, Technical University of Munich and did his Ph.D. on the numerical analysis of wind-excited movements of membrane structures in 2009. The development and validation of a virtual wind tunnel are in the center of his scientific and industrial interest since it constitutes the basis of ongoing competitiveness and innovation leadership in the field of light-weight structures. He is Partner of str.ucture $\mathrm{GmbH}$, Stuttgart.

Julian Lienhard born in 1980, completed his doctorate summa cum laude with his dissertation on bending-active structures at the Institute of Building Structures and Structural Design, University of Stuttgart in 2014. Along with a number of different design studios, he is teaching the Principles of Membrane Structures course at the University of Stuttgart, the Technical University of Vienna and since 2016 as a Visiting Professor at HCU Hamburg. His scientific interest in the principles of light-weight structures goes along with various realized experimental structures and proto-types along his academic and professional career. This forms the basis of his international structural engineering consultancy as a recognized specialist in membrane roofs and facades. Julian is Co-Founder and Managing Director of str.ucture $\mathrm{GmbH}$, Stuttgart. 